\section{New class of top-hat transformation to enhance infrared small targets}

\author{
Xiangzhi Bai, ${ }^{a}$ Fugen Zhou, ${ }^{a}$ and Yongchun Xie ${ }^{b}$ \\ ${ }^{a}$ Beijing University of Aeronautics and Astronautics, Image \\ Processing Center, Beijing, 100083, China \\ ${ }^{\mathrm{b}}$ Beijing Institute of Control Engineering, Beijing, \\ 100080, China \\ E-mail: jackybxz163@163.com
}

\begin{abstract}
To improve the performance of a top-hat transformation for infrared small target enhancement, a new class of top-hat transformation through structuring element construction and operation reorganization is proposed. The structuring element construction and operation reorganization are based on the property of the infrared small target image and thus can greatly improve the performance of small target enhancement. Experimental results verified that it was very efficient. (C) 2008 SPIE and IS\&T. [DOI: 10.1117/1.2955943]
\end{abstract}

\section{Introduction}

Top-hat transformation ${ }^{1}$ has been widely used to enhance and detect the infrared small target. ${ }^{2-5}$ But, the ability of target enhancement of top-hat transformation is weak. If the SNR of the image is low or the image has heavy clutter, then the top-hat transformation may decrease the SNR of the image, leading to losing target and increasing false alarm. One way of improving the enhancement ability of the top-hat transformation is using some preprocessing methods, such as energy cumulating ${ }^{2}$ and structuring element selection. ${ }^{3}$ Although they can perform well in some cases, these methods do not really improve the enhancement ability of the top-hat transformation. Another way is constructing the structuring elements with the purpose of the target recognition applications. ${ }^{4}$ But, the improvement of the existing method is very limited. To apparently improve the enhancement ability of the top-hat transformation, a new class of top-hat transformation through structuring element construction and operation reorganization is proposed. Experiments verified that the new top-hat transformation performed better than the classical top-hat transformation ${ }^{1-3}$ and other widely used methods for infrared small target enhancement. ${ }^{5-7}$

\section{Classical Top-Hat Transformation}

The classical white and black top-hat transformations (WTH and BTH) are defined as follows. ${ }^{1}$

$\mathrm{WTH}(x, y)=f(x, y)-f \bigcirc B(x, y)$,

$\operatorname{BTH}(x, y)=f \bullet B(x, y)-f(x, y)$,

$f \bigcirc B=(f \Theta B) \oplus B$,

Paper 08003LR received Jan. 11, 2008; revised manuscript received Apr. 16, 2008; accepted for publication May 14, 2008; published online Aug. $11,2008$.

1017-9909/2008/17(3)/030501/3/\$25.00 (C) 2008 SPIE and IS\&T. $f \bullet B=(f \oplus B) \Theta B$.

The definitions indicate that the classical top-hat transformations use of the two same structuring elements $B$ to estimate the background of the image through the target filtering by $f \bigcirc B$ or $f \bigcirc B$ and enhance the possible target regions through subtracting the estimated background from the original image.

\section{New Class of Top-Hat Transformation}

\subsection{Definitions}

The small target usually can be recognized as a small bright region embedded in the infrared clutter background. ${ }^{2}$ Thus, the gray intensity of the surrounding regions will be much different from that of the target region.

But, in classical top-hat transformation, the use of the two same structuring elements cannot differentiate the target region and the surrounding region very well through the relationship of the structuring elements, and thus, cannot utilize the gray intensity difference between the target region and the surrounding region very well for the purpose of small target enhancement. Also, because of the use of the two same structuring elements, all the pixels in the target region and the surrounding region are used for the top-hat transformation calculation. Then, the noises in these regions will affect the performance of top-hat transformation for small target enhancement, leading to losing target or increasing false alarm.

Therefore, to greatly improve the performance of top-hat transformation, the two structuring elements can be reconstructed so that the top-hat transformation can greatly suppress the effect of the noises and utilize the gray intensity difference between the target region and the surrounding region well. In light of this, a new class of top-hat transformation [new WTH (NWTH) and new BTH (NBTH)] is defined as follows:

$$
\begin{aligned}
& \operatorname{NWTH}(x, y)=f(x, y)-f \square B_{o i}(x, y), \\
& \operatorname{NBTH}(x, y)=f \square B_{o i}(x, y)-f(x, y), \\
& f \square B_{o i}(x, y)=(f \oplus \Delta B) \Theta B_{b}, \\
& f \square B_{o i}(x, y)=(f \Theta \Delta B) \oplus B_{b} .
\end{aligned}
$$

$B_{o i}$ represents two structuring elements $B_{o}$ and $B_{i}$. The relationship of the structuring elements $B_{o}, B_{i}, B_{b}$, and $\Delta B$ is demonstrated in Fig. 1, in which $O$ is the origin of all the structuring elements. $S_{\Delta}, S_{i}, S_{b}$, and $S_{o}$ are the size of the structuring elements $\Delta B, B_{i}, B_{b}$, and $B_{o}$, separately.

\subsection{Properties}

1. It can be seen from Eqs. (5) and (7) that the operation sequence of $\Theta$ and $\oplus$ in NWTH is different from that of WTH and is reorganized.In WTH, $f \bigcirc B$ started with $\Theta$ and followed by $\oplus$. According to the definitions in Ref. 1, $\Theta$ takes the small value of the corresponding region while $\oplus$ takes the large one. Thus, the negative noise in the target region and the positive noise in the surrounding region will heavily af- 


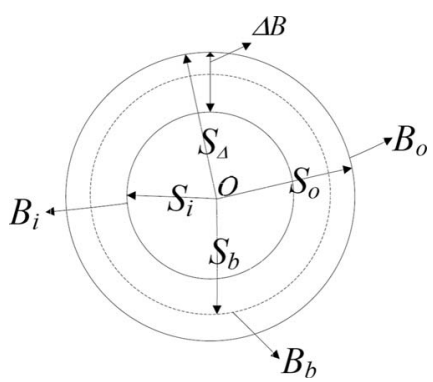

Fig. 1 Relationship of the structuring elements in NWTH and NBTH.

fect the performance of WTH. ${ }^{4}$ In NWTH, $f \boldsymbol{~} B_{o i}$ started with $\oplus$ and followed by $\Theta$. NWTH first uses $\oplus$ with $\Delta B$ to take the large value in the surrounding region and replace all the gray values of the target region with this value. Then, the following $\Theta$ replaces all the gray values of the region overlaid by $B_{b}$ with the small gray value of this region, and all the gray values of this region are from the surrounding region of the original image because of the former $\oplus$ operation. Thus, NWTH only uses the pixels of the surrounding region overlaid by $\Delta B$ for top-hat transformation calculation. Then, the noises in the target region will not affect the performance of NWTH. Also, the gray values of the target region are usually larger than the surrounding region, ${ }^{2}$ and the following $\Theta$ in NWTH replaces all the gray values of the region overlaid by $B_{b}$ with the small gray value. Thus, the positive noises in the surrounding regions will not heavily affect the performance of NWTH. Therefore, NWTH can greatly suppress the effect of the noise.

2. NWTH uses two different but related structuring elements $\Delta B$ and $B_{b}$ to replace the two same structuring elements $B$ in WTH. When NWTH is used to enhance the infrared small target, $\Delta B$ represents the surrounding region of the small target region. In this case, NWTH only uses the pixels of the surrounding region for the top-hat transformation calculation. Thus, the operation of $f-f \mathbf{m} B_{o i}$ in NWTH can well identify any difference between the surrounding re- gion and the target region. Also, the number of pixels used for calculation decreases, which decreases the probability of using noise pixels and thus suppresses the effect of the noises. These properties mean that, because of the structuring element construction and operation reorganization, NWTH can greatly suppress the effect of the noises and well use the gray intensity difference between the target region and the surrounding region in the infrared image for small target enhancement.

\section{Extension for Infrared Small Target Enhancement}

\subsection{Extension}

According to the definition of opening in WTH, the relationship between $f \bigcirc B$ and $f$ is $f \bigcirc B<f .{ }^{1}$ But, because of the operation sequence alternation of $\Theta$ and $\oplus$ in $f \mathbf{\square} B_{o i}$, the relationship between $f \mathbf{\square} B_{o i}$ and $f$ does not satisfy $f \square B_{o i}<f$ any more, which means that NWTH may produce negative gray values in the resulting image. To avoid this situation, Eq. (5) can be modified as follows:

$$
\begin{aligned}
\operatorname{NWTH}(x, y) & =\max \left[f(x, y)-f \mathbf{\square} B_{o i}(x, y), 0\right] \\
& =\max \left[f(x, y)-f \mathbf{\square} B_{o i}(x, y), f(x, y)-f(x, y)\right] \\
& =f(x, y)-\min \left[f \mathbf{\square} B_{o i}(x, y), f(x, y)\right]
\end{aligned}
$$

Let $N C_{o i}(x, y)=\min \left[f \square B_{o i}(x, y), f(x, y)\right]$, then

$\operatorname{NWTH}(x, y)=f(x, y)-N C_{o i}(x, y)$.

\subsection{Parameter Selection}

Following Fig. 1, smaller $S_{o}-S_{i}$ results in fewer pixels in $\Delta B$ being used for calculation. This will suppress the effect of the noise. Thus, smaller $S_{0}-S_{i}$ is better for the purpose of infrared small target enhancement. Also, bigger $S_{b}$ results in better filter ability of morphological operations. ${ }^{1}$ The infrared small target usually can be recognized as one protruding region embedded in the infrared image. Then, the good filter ability of $f B_{o i}$ results in good performance of target filtering and, thus, a good performance of target enhancement of NWTH. Hence, $S_{b}$ should be a large value.

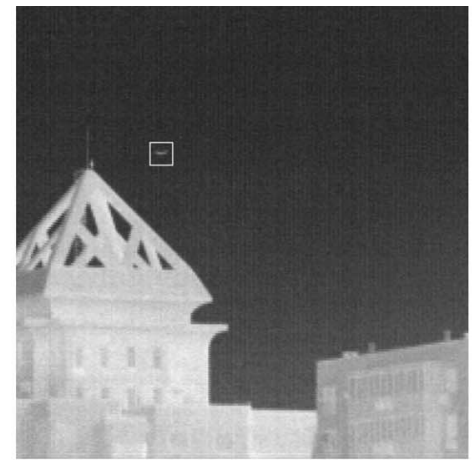

(a)

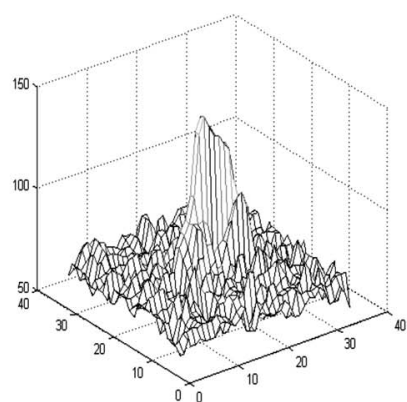

(b)

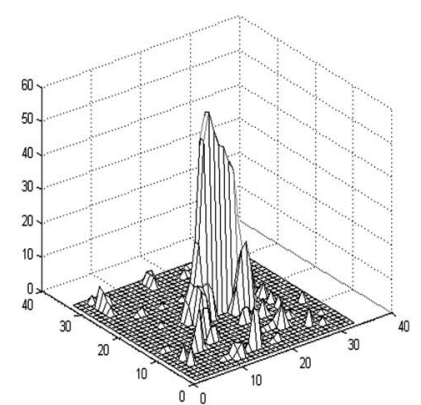

(c)

Fig. 2 Infrared small target enhancement: (a) the original image; (b) 3-D plot of the gray intensity of the target region and the surrounding region in (a); and (c) 3-D plot of the gray intensity of the target region and the surrounding region after NWTH. 
Table 1 Comparison of target enhancement (LSBR).

\begin{tabular}{ccccc}
\hline \hline & Target 1 & Target 2 & Target 3 & Target 4 \\
\hline Original image & 0.8532 & 1.4837 & 3.6623 & 2.2195 \\
Max-median $(3 \times 3)$ & 6.1695 & 7.2690 & 8.7318 & 8.0548 \\
Max-mean $(3 \times 3)$ & 1.6475 & 2.4020 & 3.8206 & 3.1018 \\
Max-median $(5 \times 5)$ & 1.3856 & 1.8652 & 3.8200 & 2.2526 \\
Max-mean $(5 \times 5)$ & 3.0128 & 4.3528 & 6.3965 & 5.4294 \\
WTH & 1.6353 & 2.7166 & 4.8352 & 3.8702 \\
U-kernel & 2.5590 & 3.8599 & 7.3865 & 5.0803 \\
E-kernel & 2.3663 & 3.5784 & 6.9313 & 4.7006 \\
NWTH & 7.2048 & 9.6613 & 11.3095 & 11.6462 \\
\hline \hline
\end{tabular}

Therefore, to make NWTH perform well on the application of infrared small target enhancement, some parameters can be defined as follows: $S_{o}-S_{i}=1, S_{b}=S_{o}$.

The infrared small target usually has an irregular shape with small size; thus, the shape of the structuring elements can usually be a circle or rectangle. The shape of the structuring elements used in this paper is a rectangle.

Obviously, when $S_{b}=S_{i}$, expression (5) becomes the tophat transformation proposed in Ref. 4, which is a specific case of Eq. (5). But, following the analysis above, Ref. 4 will result in negative gray value in the resulting image and is not very efficient with $S_{b}=S_{i}$ for infrared small target enhancement.

\section{Experimental Results}

To verify the performance of NWTH for infrared small target enhancement, some infrared images with size 720 $\times 576$ obtained from the forward-looking infrared (FLIR) system are used. One image that is cut from the original image with an appropriate size is demonstrated in Fig. 2(a). The target is an aircraft and is labeled by a rectangle. In Fig. 2(a) the noises and clutter are heavy. In addition, the target is dim as illustrated in the 3-D plot of the gray intensity [Fig. 2(b)]. But, NWTH can greatly enhance the small target as shown in Fig. 2(c). In Fig. 2(c), the noises and clutter are well suppressed and the target is much enhanced, leading to easy detection of the target.

To prove the improved performance of NWTH for infrared small target enhancement, some widely used methods [U-kernel, ${ }^{7}$ E-kernel, ${ }^{7}$ Max-mean, ${ }^{6}$ Max-median, ${ }^{6}$ WTH (Refs. 1-3)] as well as NWTH are applied on the images. The measure local signal to background ratio ${ }^{5}$ (LSBR) is adopted here to compare their performances, and the result is shown in Table 1,

$\mathrm{LSBR}=10 \log \left\{\frac{1}{\sigma_{b}^{2}} \sum_{k=-W / 2}^{W / 2} \sum_{j=-W / 2}^{W / 2}\left[f(s-k, r-j)-m_{b}\right]\right\}$

where $\sigma_{b}^{2}$ and $m_{b}$ are the variance and mean of the background in the window described by the width and height $W$

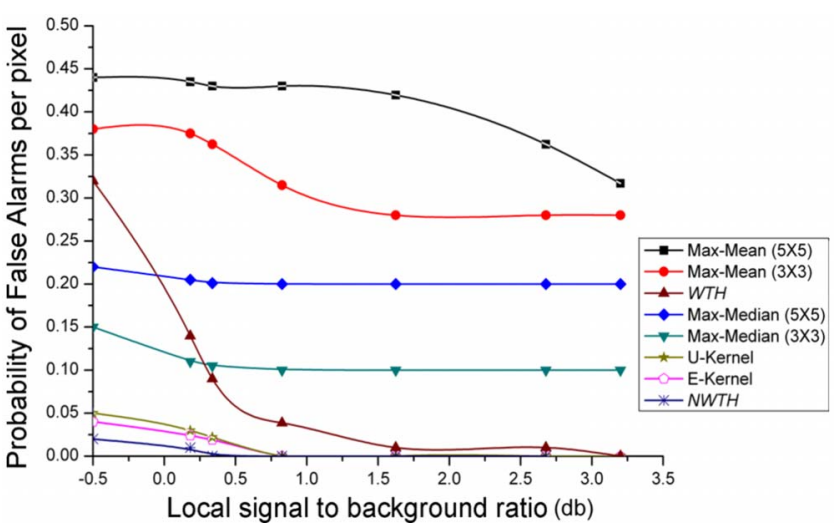

Fig. 3 PFA-LSBR curves.

around the interest pixel $(s, r)$, respectively. To calculate valid LSBR value, $W$ should be much larger than the size of the infrared small target. Here, $W=33$. Larger LSBR means better performance of target enhancement. In Table 1, because of the heavy noise and clutter of the images, the LSBRs of WTH are worse than most of the other methods. Conversely, the performances of NWTH are much better than other methods no matter how small the LSBR of the original image is.

Another measure named probability of false alarms (PFA) per pixel versus the input LSBR (Ref. 5) is also adopted to describe the performances of the methods, and the result is shown in Fig. 3. In Fig. 3, the number of false alarms caused by NWTH is much smaller than other methods at any LSBR, which also verifies that the performance of NWTH is efficient.

\section{Conclusions}

A new class of top-hat transformation through structuring element construction and operation reorganization is proposed to improve the performance of top-hat transformation for infrared small targe enhancement. The experiments verify that the new class of top-hat transformation can be well used for infrared small target enhancement and performs better than other widely used methods. Moreover, the appropriate selection of $B_{i}, B_{b}$, and $B_{o}$ will result in good performance of NWTH and NBTH for different application purposes.

\section{References}

1. J. Serra, Image Analysis and Mathematical Morphology, Academic Press, New York (1982).

2. F. Zhang, C. Li, and L. Shi, "Detecting and tracking dim moving point target in IR image sequences," Infrared Phys. Technol. 46, 323328 (2005)

3. M. Zeng, J. Li, and Z. Peng, "The design of top-hat morphological filter and application to infrared target detection," Infrared Phys. Technol. 48, 67-76 (2006).

4. P. T. Jackway, "Improved morphological top-hat," Electron. Lett., 36(14), 1194-1195 (2000).

5. T. Soni, J. R. Zeidler, and W. H. Ku, "Performance evaluation of 2-D adaptive prediction filters for detection of small objects in image data," IEEE Trans. Image Process. 2(3), 327-340 (1993).

6. S. D. Deshpande, M. H. Er, V. Ronda, and P. Chan, "Max-mean and max-median filters for detection of small-targets," Proc. SPIE 3809 74-83 (1999).

7. S. Leonov, "Nonparametric method for clutter removal," IEEE Trans. Aerosp. Electron. Syst. 37(3), 832-848 (2001). 